COSMIC RAYS

\section{More about Muons}

from a Correspondent

"THE possibility of a consistent explanation of various phenomena involving cosmic ray muons" is the intriguing title of a recent article by the Polish authors Wdowczyk and Zujewska ( $J$. Phys. A., 5, 1514; 1972). As the title implies several recent cosmic ray observations have yielded results not readily explainable by accepted theories. Among these phenomena are the angular distribution of muon-poor air showers, the high energy muon spectrum derived from the burst size spectrum and the angular distribution of energetic muons observed by a group at the University of Utah.

The angular distribution of extensive air showers has been measured at large zenith angles $\left(>60^{\circ}\right)$ by Catz et al. (Proc. Twelfth Inter. Conf. on Cosmic Rays, Hobart, 1030 ; 1972). They found many more showers than normal theories predict, and also that these showers were lacking in muons. The high energy muon flux at sea level derived from observation of the spectrum of burst sizes yields a flux higher than other methods, for example underground intensity measurements (see Chin et al., Proc. Eleventh Inter. Conf. on Cosmic Rays, Budapest, 65 ; 1969).

Even stronger evidence that something was wrong seemed to come from the work of Keuffel et al. (Proc. Eleventh Inter. Conf. on Cosmic Rays, Budapest, $183 ; 1969)$. In the conventional picture of high energy interactions the dominant source of muons is the decay of pions and kaons. The competition between the decay and interaction of these parent particles should produce an enhancement of muon intensity at large zenith angles, but the Utah group have presented experimental evidence that above muon energies of $10^{12} \mathrm{eV}$ this enhancement does not occur.

Wdowczyk and Zujewska attempt to explain these observations in terms of the presence of a partly passive, long lived, heavy particle (the X-particle) that is produced in the interactions of primary protons with air nuclei. Such a hypothesis has indeed been proposed previously to explain some of the effects. In order to bring some consistency into the explanations, however, the authors propose detailed properties for these Xparticles. The production spectrum of X-particles above about $10^{12} \mathrm{eV}$ is taken as parallel to the primary particle spectrum but with an intensity lower by a factor of 2,000 . The lower limit to the cross-section for particle production is taken to be about $0.2 \mathrm{mb}$ on the assumption that the $\mathrm{X}$-particles are produced in pairs with the total energy of the pair half the primary energy.
Wdowczyk and Zujewska use the angular distribution of the muon-poor showers to give the attenuation length in the interval 1,000 to $2,500 \mathrm{GeV}$. The inelasticity coefficient is taken as 0.1 and the interaction length as $400 \mathrm{~g}$ $\mathrm{cm}^{-2}$. The dominant decay mode is assumed to be $\mathrm{X}^{\circ} \rightarrow \mu^{+}+\mu^{-}$with a lifetime between $10^{-7}$ and $10^{-8} \mathrm{~s}$, a limit set by the fact that the particle has not been observed in accelerator experiments but has to be consistent with the onset of the "Utah effect".

The model succeeds in predicting a sea-level muon energy spectrum consistent with experimental data. The muon spectra obtained from burst measurements are excluded, for on the basis of the model the bursts would be caused partly by interaction of muons and partly by the direct interactions of the X-particles. Wdowczyk and Zujewska also consider the burst size spectrum as measured, and show that this is in better agreement with expectations when the contribution from both muons and $\mathrm{X}$-particles is taken into account.

The angular dependence of the in- tensity of high energy muons is taken as a superposition of the angular variation of the intensity of muons produced by decaying pions and the variation of muons due to the X-process. By choosing the $\mathrm{X}$-particle properties as detailed, an explanation of the Utah result is obtained. The authors also suggest that other cosmic ray phenomena, the presence of muon bundles in extensive air shows for example, can be explained on the basis of the same hypothesis.

It should, however, be pointed out that both the Utah effect and the presence of muon bundles have very recently been called into question by those who observed them. In neither case are the experimenters any longer prepared to stand resolutely by their previous results. The experimental backing for the X-particle hypothesis has therefore already been considerably weakened. Further experiments, based perhaps on the suggestions of Wdowczyk and Zujewska, have to be performed before confirmation (or denial) of the X-particle theory can be claimed.

\title{
Finite Elements for Alluvial Valleys
}

Mathematical understanding of the local effects of seismic waves is made difficult because of variations in structure and topography. Earth scientists are thus forced to oversimplify geological structures in any theoretical work, although numerical analysis is now yielding useful approaches to problems which theoreticians have hitherto regarded as insoluble. The finite element method, for example, has recently opened up new possibilities. This method was developed originally to solve stress-strain problems in aeronautical engineering but it has also proved useful in, for example, structural geology, dam engineering, rock mechanics and studies of tidal loading. In next Monday's Nature Physical Science (December 4), L. A. Drake describes an application of the method to the study of surface wave propagation.

Drake took an elastic finite element model of a valley in which a veneer of alluvium overlay a basin of shale and basement rocks. He used the model to look at the amplitude variation of a Rayleigh wave with a period of $2 \mathrm{~s}$ and found that the amplitude of the maximum ground motion was amplified by factors of about 270 and 35 in the horizontal and vertical components respectively. This result confirmed previous work by Mooney and Bolt (Bull. Seism. Soc. Amer., 56, 43; 1966), but the studies of these authors related to a plain layered structure only, whereas Drake was able to study the distribution of the ground motion across a geological structure. He found that the maximum amplitude was offset from the centre of the valley. By varying the contact angle between the shale and basement no significant change was observed but the amplification of the horizontal component was sensitive to the lateral extent of the alluvial deposit. If the alluvium is deposited over a restricted area the amplification factor would be reduced.

Drake's use of the finite element method to look at the local effects of seismic wave propagation has many applications. The alluvial valleys of earthquake belts are frequently areas where the greatest damage to property and loss of life occurs in the event of a large earthquake. Even at considerable distances from the epicentre liquefaction, fracture, subsidence and landslips in unconsolidated material can occur; it should now be possible to predict more closely than before the effects of seismic disturbance.

The chief problems in applying Drake's technique to real structures are that geological models are difficult to obtain, that ground water may have significant effects and that the physical parameters of the rocks may be anisotropic and highly variable. It is, however, possible to place many of these factors into the finite element models: some will have little effect but others may be of great importance. Drake's models are a good first-order approximation and form a basis for further work which may help to reduce the risk of disaster in the seișmically active areas of the world. 